\title{
Trends in acute ischemic stroke treatments and mortality in the United States from 2012 to 2018
}

\author{
*Sharath Kumar Anand, BS, ${ }^{1}$ William J. Benjamin IV, MPH, ${ }^{2}$ Arjun Rohit Adapa, MS, ${ }^{2}$ \\ Jiwon V. Park, BS, ${ }^{2}$ D. Andrew Wilkinson, MD, ${ }^{1,3}$ Badih J. Daou, MD, ${ }^{1}$ James F. Burke, MD, ${ }^{4}$ and \\ Aditya S. Pandey, MD'
}

\begin{abstract}
${ }^{1}$ Department of Neurosurgery, ${ }^{2}$ School of Medicine, and ${ }^{4}$ Department of Neurology, University of Michigan, Ann Arbor, Michigan; and ${ }^{3}$ Department of Neurosurgery, Penn State Health, Hershey, Pennsylvania
\end{abstract}

\begin{abstract}
OBJECTIVE The establishment of mechanical thrombectomy (MT) as a first-line treatment for select patients with acute ischemic stroke (AIS) and the expansion of stroke systems of care have been major advancements in the care of patients with AIS. In this study, the authors aimed to identify temporal trends in the usage of tissue-type plasminogen activator (tPA) and MT within the AIS population from 2012 to 2018, and the relationship to mortality.
\end{abstract}

METHODS Using a nationwide private health insurance database, 117,834 patients who presented with a primary AIS between 2012 and 2018 in the United States were identified. The authors evaluated temporal trends in tPA and MT usage and clinical outcomes stratified by treatment and age using descriptive statistics.

RESULTS Among patients presenting with AIS in this population, the mean age was 69.1 years (SD \pm 12.3 years), and $51.7 \%$ were female. Between 2012 and 2018, the use of tPA and MT increased significantly (tPA, $6.3 \%$ to $11.8 \%, p<$ $0.0001 ; \mathrm{MT}, 1.6 \%$ to $5.7 \%, \mathrm{p}<0.0001)$. Mortality at 90 days decreased significantly in the overall AIS population $(8.7 \%$ to $6.7 \%, p<0.0001)$. The largest reduction in 90 -day mortality was seen in patients treated with MT $(21.4 \%$ to $14.1 \%, p=$ $0.0414)$ versus tPA $(11.8 \%$ to $7.0 \%, p<0.0001)$ versus no treatment $(8.3 \%$ to $6.3 \%, p<0.0001)$. Age-standardized mortality at 90 days decreased significantly only in patients aged $71-80$ years $(11.4 \%$ to $7.8 \%, p<0.0001)$ and $>81$ years $(17.8 \%$ to $11.6 \%, p<0.0001)$. Mortality at 90 days stagnated in patients aged 18 to 50 years $(3.0 \%$ to $2.2 \%, p=0.4919)$, 51 to 60 years (3.8\% to $3.9 \%, p=0.7632)$, and 61 to 70 years $(5.5 \%$ to $5.2 \%, p=0.2448)$.

CONCLUSIONS From 2012 to 2018, use of tPA and MT increased significantly, irrespective of age, while mortality decreased in the entire AIS population. The most dramatic decrease in mortality was seen in the MT-treated population. Age-standardized mortality improved only in patients older than 70 years, with no change in younger patients.

https://thejns.org/doi/abs/10.3171/2021.4.FOCUS21117

KEYWORDS ischemic stroke; tissue-type plasminogen activator; thrombectomy; mortality; trends

$\mathrm{S}$ TROKE is the second leading cause of mortality worldwide, ${ }^{1}$ and the fifth leading cause of mortality in the United States. ${ }^{2}$ For 20 years, intravenous (IV) tissuetype plasminogen activator (tPA) was the primary treatment for acute ischemic stroke (AIS), ${ }^{3-5}$ until 2015, when use of mechanical thrombectomy (MT) demonstrated considerably improved outcomes in select patients with AIS. ${ }^{6-}$ ${ }^{11}$ Despite the proven efficacy of these treatments, IV tPA and MT remain severely underutilized. ${ }^{12,13}$ To increase utilization of these therapies and standardize AIS care across the US, primary and comprehensive stroke centers (CSCs) were established as system-based strategies to improve outcomes. The first CSC certifications approved in 2012 required, among other things, high volumes, advanced imaging techniques, dedicated neurological intensive care units, and after-hospital care coordination for patients. ${ }^{14}$

These developments in AIS treatment and organization have proven beneficial at the individual level and have shown significant negative trends in mortality at the population level, ${ }^{15,16}$ although existing studies predate the expansion of the MT window to 24 hours for select patients. Similarly, various studies have analyzed AIS treatment and outcome trends over time using large national databases. ${ }^{15,17-21}$ However, few studies have reported outcomes

ABBREVIATIONS AIS = acute ischemic stroke; CPT = Current Procedural Terminology; CSC = comprehensive stroke center; $\mathrm{HT}=$ hemorrhagic transformation; ICD-9 = International Classification of Diseases, Ninth Revision; ICD-10 = International Classification of Diseases, Tenth Revision; IV = intravenous; MCE = malignant cerebral edema; $\mathrm{mRS}=$ modified Rankin Scale; $\mathrm{MT}=$ mechanical thrombectomy; $\mathrm{PSC}=$ primary stroke center; $\mathrm{tPA}=$ tissue-type plasminogen activator.

SUBMITTED February 28, 2021. ACCEPTED April 7, 2021

INCLUDE WHEN CITING DOI: 10.3171/2021.4.FOCUS21117.

* S.K.A. and W.J.B. contributed equally to this work. 
following specific treatments. ${ }^{22-24}$ The objective of this study was to investigate trends in IV tPA and MT usage and associated patient outcomes after the major MT trials among participants in a large, national, private health insurance database.

\section{Methods \\ Data Source}

Using de-identified claims from a large private healthcare network in the US (Clinformatics Data Mart database, Optum Inc.), 117,834 beneficiaries who presented with a primary ischemic stroke between 2012 and 2018 were identified. Patient data were reported by medical claims, facility details, and inpatient summaries. Diagnoses were identified using International Classification of Diseases, Ninth and Tenth Revisions (ICD-9 and ICD10). Procedures were identified using Current Procedural Terminology (CPT) codes alongside ICD-9 and ICD-10 procedure codes. The database does not include patient identifiers and was deemed exempt from Institutional Review Board approval.

\section{Patient Selection}

We queried the database for adult patient records with a primary diagnosis of ischemic stroke between 2012 and 2018 and defined by ICD-9 and ICD-10 codes. ${ }^{25}$ ICD-9 codes were used for records from 2012 to mid-2015, and ICD-10 codes were used for records from mid-2015 to 2018. The year 2018 was the most recent year available for our analyses.

\section{Variables}

Patient-level variables included age at diagnosis; sex; race; discharge status; and 30-day, 60-day, 90-day, and 6-month mortality. For demographic variables, missing values were reported as unknown. Computed procedural variables were coded using either CPT codes or ICD-9 and ICD-10 procedural codes and are shown in Supplemental Table 1. We used standardized conversion charts to convert ICD-9 to ICD-10.

Discharge disposition was recorded according to discharge code as specified in the data set. For purposes of analysis, the top 5 most common dispositions (home with self-care, home with a service organization, inpatient rehabilitation, skilled nursing facility, and transfer to a short-term hospital) were represented individually, while an "other" group included all other dispositions, such as intermediate care facility, other type of institution for inpatient care, left against medical advice, admitted as inpatient to this hospital, still inpatient expecting to return as outpatient or status yet to be defined at the state level, transfer to a federal healthcare facility, hospice (home or medical), transfer within the institution, and transfer to a long-term hospital, nursing home facility certified under Medicare, psychiatric hospital, critical access hospital, or another type of healthcare facility.

We also analyzed the relationship of hospital size to discharge disposition and mortality for all patients with AIS, those who underwent thrombectomy, and those who received tPA treatment. Hospital size was categorized into large ( $\geq 250$ beds), medium (100-249 beds), and small ( $\leq$ 100 beds).

\section{Statistical Analysis}

All statistical analyses were completed using SAS software version 9.4 (SAS Institute Inc.). Statistical significance was defined as $\mathrm{p}<0.05$ and 2-tailed tests were used. Excel (Microsoft) was used to plot figures.

General characteristics of the stroke cohort were assessed using yearly frequency tables, including overall trends in MT, IV tPA, both MT and IV tPA, neither MT nor IV tPA, malignant cerebral edema (MCE), hemorrhagic transformation (HT), discharge disposition, and mortality; treatment-specific outcomes in MCE, HT, discharge disposition, and mortality; and trends in MT, IV tPA, both MT and IV tPA, neither MT nor IV tPA, MCE, HT, discharge disposition, and mortality stratified by age. Cochran-Armitage testing was used to assess for trends, and the frequency procedure was used to build frequency tables.

\section{Results}

\section{Overall Cohort}

The number of active enrollees within the database slightly increased from 15,624,321 patients in 2012 to $20,145,678$ patients in 2018 . The overall mortality rate followed a similar trend from $0.5 \%$ in 2012 to $0.6 \%$ in 2018 . Our criteria identified 117,834 patients from 2012 to 2018 with a primary diagnosis of AIS and a mean age of 69.1 with a standard deviation \pm 12.3 years; $51.7 \%$ were female (Table 1). The yearly percentage of all enrollees hospitalized with a primary diagnosis of AIS in the entire cohort averaged $0.8 \%$ and ranged from $0.8 \%$ in 2012 to $0.9 \%$ in 2018.

\section{Treatment of AIS}

Among 117,834 AIS admissions, 4155 patients (3.5\%) received MT, 10,976 patients $(9.3 \%)$ received IV tPA, and 1203 patients $(1.0 \%)$ received both. When comparing treatment modalities in 2012 with 2018, significant increases were noted in the proportional usage of MT (1.6\% to $5.7 \%, \mathrm{p}<0.0001)$, IV tPA $(6.3 \%$ to $11.8 \%$, p < $0.0001)$, and combined MT and IV tPA $(0.7 \%$ to $1.7 \%, \mathrm{p}<$ 0.0001) (Fig. 1 and Table 2). Of special interest, the largest increase in MT rate occurred between 2017 and 2018 (4.3\% to $5.7 \%$ ), followed by 2014 and 2015 (1.7\% to $2.8 \%$ ).

\section{Outcomes of AIS}

Between 2012 and 2018, 30-day, 60-day, 90-day, and 6-month mortality rates in patients with AIS all significantly decreased (4.8\% to $4.2 \%, \mathrm{p}<0.0001 ; 7.7 \%$ to $6.0 \%$, $\mathrm{p}=0.0002 ; 8.7 \%$ to $6.7 \%, \mathrm{p}<0.0001 ;$ and $10.3 \%$ to $7.8 \%$, $\mathrm{p}<0.0001$, respectively). There were significant decreases in the rates of routine discharge home (41.3\% to $37.3 \%, \mathrm{p}$ $<0.001)$ and transfer to another short-term hospital $(7.2 \%$ to $5.2 \%, p<0.001$ ), and there were significant increases in the rates of discharge home under a service organization (10.4\% to $12.8 \%, \mathrm{p}<0.0001)$ and inpatient rehabilitation ( $8.9 \%$ to $13.2 \%, \mathrm{p}<0.0001$ ). Lastly, the rates of discharge 
TABLE 1. Overall demographics of privately insured patients with AIS between 2012 and 2018

\begin{tabular}{|c|c|c|c|c|c|c|c|}
\hline \multirow[b]{2}{*}{ Characteristic } & \multicolumn{7}{|c|}{ No. of Patients (\%) } \\
\hline & $2012(n=12,831)$ & $2013(n=13,646)$ & $2014(n=13,898)$ & $2015(n=15,474)$ & $2016(n=18,227)$ & $2017(n=21,826)$ & $2018(n=21,932)$ \\
\hline \multicolumn{8}{|l|}{ Age, yrs } \\
\hline $18-50$ & $1,206(9.4)$ & $1,211(8.9)$ & $1,261(9.1)$ & $1,443(9.3)$ & $1,498(8.2)$ & $1,625(7.5)$ & $1,647(7.5)$ \\
\hline $51-60$ & $1,618(12.6)$ & $1,727(12.7)$ & $1,586(11.4)$ & $1,875(12.1)$ & 2,198 (12.1) & $2,409(11.0)$ & $2,439(11.1)$ \\
\hline $61-70$ & $3,247(25.3)$ & $3,659(26.8)$ & $3,713(26.7)$ & $4,255(27.5)$ & $5,286(29.0)$ & $6,262(28.7)$ & 6,468 (29.5) \\
\hline $71-80$ & $4,697(36.6)$ & $4,806(35.2)$ & $4,741(34.1)$ & $5,062(32.7)$ & $5,872(32.2)$ & $6,728(30.8)$ & $6,693(30.5)$ \\
\hline$\geq 81$ & $1,722(13.4)$ & $1,867(13.7)$ & $2,325(16.7)$ & $2,585(16.7)$ & $3,043(16.7)$ & $4,410(20.2)$ & $4,249(19.4)$ \\
\hline Unknown & $341(2.7)$ & $376(2.8)$ & $272(2.0)$ & $254(1.6)$ & $330(1.8)$ & $392(1.8)$ & $436(2.0)$ \\
\hline \multicolumn{8}{|l|}{ Sex $^{*}$} \\
\hline$F$ & $6,451(51.6)$ & $6,847(51.6)$ & $7,089(52.0)$ & $8,045(52.8)$ & $9,552(53.3)$ & $11,452(53.4)$ & $11,435(53.2)$ \\
\hline $\mathrm{M}$ & $6,050(48.4)$ & $6,430(48.4)$ & $6,551(48.0)$ & $7,185(47.2)$ & $8,360(46.7)$ & $9,990(46.6)$ & $10,070(46.8)$ \\
\hline \multicolumn{8}{|l|}{ Race } \\
\hline Asian & $339(2.6)$ & $330(2.4)$ & $367(2.6)$ & $426(2.8)$ & $399(2.2)$ & $460(2.1)$ & $405(1.9)$ \\
\hline Black & $1,702(13.3)$ & $1,785(13.1)$ & $1,847(13.3)$ & $1,997(12.9)$ & $2,956(16.2)$ & $3,187(14.6)$ & $2,851(13.0)$ \\
\hline Hispanic & $1,025(8.0)$ & $1,076(7.9)$ & $1,232(8.9)$ & $1,333(8.6)$ & $1,451(8.0)$ & $1,806(8.3)$ & $1,569(7.2)$ \\
\hline White & $8,394(65.4)$ & 9,033 (66.2) & $9,115(65.6)$ & $10,142(65.5)$ & $11,370(62.4)$ & $13,049(59.8)$ & $12,116(55.2)$ \\
\hline Unknown & $1,371(10.7)$ & $1,422(10.4)$ & $1,337(9.6)$ & $1,576(10.2)$ & $2,051(11.3)$ & $3,324(15.2)$ & $4,991(22.8)$ \\
\hline
\end{tabular}

* Sex data were missing for 2,327 patients.

to skilled nursing facilities $(20.2 \%$ to $19.6 \%, \mathrm{p}=0.4403)$ and discharge to "other" $(12.0 \%$ to $11.9 \%, \mathrm{p}=0.2012) \mathrm{re}$ mained steady (Table 2).

\section{Outcomes of AIS by Treatment Modality}

The percentage of patients discharged home with a service organization significantly increased irrespective of treatment, with the most dramatic increase seen in patients who underwent MT (2.0\% to $8.5 \%, \mathrm{p}=0.0001)$ versus IV tPA $(6.4 \%$ to $12.6 \%, \mathrm{p}<0.0001)$ versus both MT and IV tPA $(3.6 \%$ to $9.6 \%, \mathrm{p}=0.0108)$ versus neither treatment (10.8\% to $13.1 \%$, p < 0.0001). Similarly, the MT cohort had the most dramatic decline in mortality, specifically at 30 days $(16.4 \%$ to $9.0 \%, \mathrm{p}=0.0415), 60$ days $(21.4 \%$ to $13.2 \%, \mathrm{p}=0.0144), 90$ days $(21.4 \%$ to $14.1 \%, \mathrm{p}=0.0414)$, and 6 months $(22.4 \%$ to $15.4 \%, \mathrm{p}=0.0106)$. Also of note, transfer to another short-term hospital significantly decreased in the cohorts treated with MT $(13.9 \%$ to $8.6 \%, \mathrm{p}$ $=0.0005)$, both tPA and MT $(14.3 \%$ to $7.4 \%, \mathrm{p}=0.0001)$, and neither tPA nor MT $(6.9 \%$ to $4.9 \%, \mathrm{p}<0.0001)$, and trended toward significance in the tPA-only cohort $(10.4 \%$ to $5.2 \%, \mathrm{p}=0.0878$ ) (Fig. 2 and Table 3 ).

\section{Outcomes of AIS by Age}

Between 2012 and 2018, the proportional use of MT and IV tPA significantly increased across all age groups. Similarly, discharge to inpatient rehabilitation facilities significantly increased across all age groups. Routine discharge home decreased in all age groups except septuagenarians, and transfer to another short-term hospital decreased in all age groups other than those younger than 50 years. Contrarily, discharge home with a service organization increased in all age groups except sexagenarians. Among patients 18 to 70 years of age, there were no significant trends in mortality due to stroke at 30,60 , or 90 days or 6 months. Only in patients 71 years of age and older did mortality decrease at all time points (Fig. 3 and Supplemental Table 2).

\section{Outcomes of AIS by Hospital Size}

Among all patients with AIS, 30-day mortality was lowest for medium-sized health systems (3.96\%); however, the greatest absolute difference was only $0.53 \%$ when compared with the highest mortality rate in the large-sized health systems (4.49\%). The discharge disposition of the entire AIS population was more frequently to home when patients were managed at large and medium health systems (39.9\% and 39.89\%) compared with small health systems $(36.58 \%, \mathrm{p}<0.0001)$. Among patients with AIS who underwent MT, 30-day mortality was higher in small health centers compared with medium and large centers $(22.58 \%$ vs $9.77 \%$ vs $9.90 \%, \mathrm{p}=0.0637)$, and the

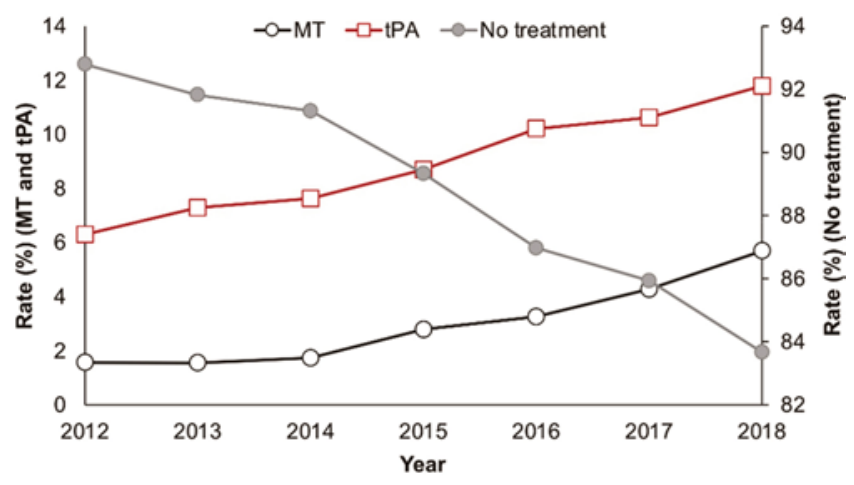

FIG. 1. Trends in AIS treatment, including MT, tPA, and no treatment. 
TABLE 2. Trends in treatments and outcomes of patients with AIS between 2012 and 2018

\begin{tabular}{|c|c|c|c|c|c|c|c|c|}
\hline \multirow[b]{2}{*}{ Characteristic } & \multicolumn{7}{|c|}{ No. of Patients (\%) } & \multirow[b]{2}{*}{$p$ Value } \\
\hline & $\begin{array}{c}2012 \\
(n=12,831)\end{array}$ & $\begin{array}{c}2013 \\
(n=13,646)\end{array}$ & $\begin{array}{c}2014 \\
(n=13,898)\end{array}$ & $\begin{array}{c}2015 \\
(n=15,474)\end{array}$ & $\begin{array}{c}2016 \\
(n=18,227)\end{array}$ & $\begin{array}{c}2017 \\
(n=21,826)\end{array}$ & $\begin{array}{c}2018 \\
(n=21,932)\end{array}$ & \\
\hline MT & $201(1.6)$ & $212(1.6)$ & $242(1.7)$ & $432(2.8)$ & $594(3.3)$ & $934(4.3)$ & $1,248(5.7)$ & $<0.0001$ \\
\hline IV tPA & $808(6.3)$ & $995(7.3)$ & $1,060(7.6)$ & $1,347(8.7)$ & $1,861(10.2)$ & $2,319(10.6)$ & $2,586(11.8)$ & $<0.0001$ \\
\hline IV tPA \& MT & $84(0.7)$ & $91(0.7)$ & $94(0.7)$ & $147(1.0)$ & $167(0.9)$ & $255(1.2)$ & $365(1.7)$ & $<0.0001$ \\
\hline Neither MT nor tPA & $11,906(92.8)$ & $12,530(91.8)$ & $12,690(91.3)$ & $13,823(89.3)$ & $15,852(87.0)$ & $18,755(85.9)$ & $18,350(83.7)$ & $<0.0001$ \\
\hline $\begin{array}{l}\text { Decompressive } \\
\text { hemicraniectomy }\end{array}$ & $40(0.3)$ & $47(0.3)$ & $47(0.3)$ & $58(0.4)$ & $59(0.3)$ & $61(0.3)$ & $62(0.3)$ & 0.2283 \\
\hline MCE & $496(3.9)$ & $587(4.3)$ & $668(4.8)$ & $775(5.0)$ & $970(5.3)$ & $1,238(5.7)$ & $1,415(6.5)$ & $<0.0001$ \\
\hline HT & $677(5.3)$ & $764(5.6)$ & $788(5.7)$ & $867(5.6)$ & $1,016(5.6)$ & $1,326(6.1)$ & $1,503(6.9)$ & $<0.0001$ \\
\hline \multicolumn{9}{|l|}{ Discharge disposition } \\
\hline Home routine & $5,306(41.4)$ & $5,552(40.7)$ & $5,459(39.3)$ & $6,127(39.6)$ & $7,064(38.8)$ & $8,233(37.7)$ & $8,180(37.3)$ & $<0.0001$ \\
\hline Home health & $1,333(10.4)$ & $1,545(11.3)$ & $1,526(11.0)$ & $1,624(10.5)$ & $2,118(11.6)$ & $2,703(12.4)$ & $2,811(12.8)$ & $<0.0001$ \\
\hline Skilled nursing facility & $2,594(20.2)$ & $2,664(19.5)$ & $2,890(20.8)$ & $3,067(19.8)$ & $3,586(19.7)$ & $4,442(20.4)$ & $4,303(19.6)$ & 0.4403 \\
\hline $\begin{array}{l}\text { Transfer to short-term } \\
\text { hospital }\end{array}$ & $919(7.2)$ & $884(6.5)$ & $859(6.2)$ & $1,012(6.5)$ & $1,063(5.8)$ & $1,128(5.2)$ & $1,132(5.2)$ & $<0.0001$ \\
\hline Inpatient rehabilitation & $1,138(8.9)$ & $1,306(9.6)$ & $1,464(10.5)$ & $1,775(11.5)$ & $2,237(12.3)$ & $2,704(12.4)$ & $2,901(13.2)$ & $<0.0001$ \\
\hline Other† & $1,541(12.0)$ & $1,695(12.4)$ & $1,700(12.2)$ & $1,869(12.1)$ & $2,159(11.9)$ & $2,616(12.0)$ & $2,605(11.9)$ & 0.2012 \\
\hline \multicolumn{9}{|l|}{ Mortality } \\
\hline 30-day & $618(4.8)$ & $582(4.3)$ & $663(4.8)$ & $756(4.9)$ & $730(4.0)$ & $887(4.1)$ & $916(4.2)$ & $<0.0001$ \\
\hline 60-day & $982(7.7)$ & $918(6.7)$ & $1,015(7.3)$ & $1,137(7.4)$ & $1,197(6.6)$ & $1,376(6.3)$ & $1,318(6.0)$ & 0.0002 \\
\hline 90-day & $1,117(8.7)$ & $1,021(7.5)$ & $1,136(8.2)$ & $1,285(8.3)$ & $1,348(7.4)$ & $1,532(7.0)$ & $1,475(6.7)$ & $<0.0001$ \\
\hline 6-mo & $1,321(10.3)$ & $1,212(8.9)$ & $1,357(9.8)$ & $1,507(9.7)$ & $1,600(8.8)$ & $1,840(8.4)$ & $1,700(7.8)$ & $<0.0001$ \\
\hline
\end{tabular}

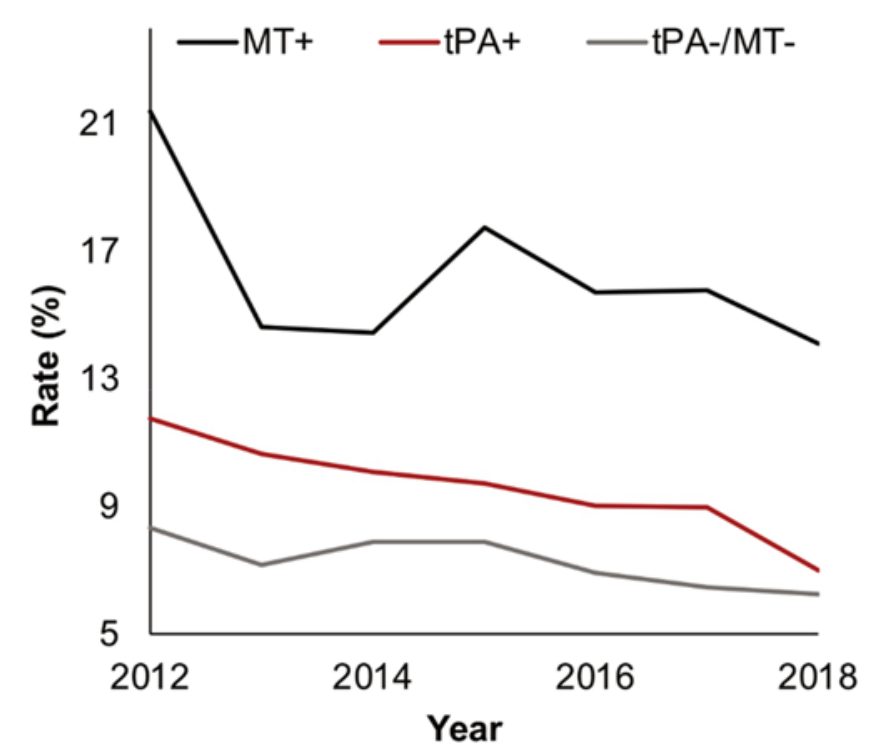

FIG. 2. Treatment-standardized 90-day mortality. discharge disposition to home was lowest for small health systems compared with medium and large centers $(9.68 \%$ vs $24.31 \%$ vs $21.76 \%, p=0.1299)$.

Among patients with AIS who received IV tPA, 30-day mortality (small $5.38 \%$ vs medium $5.57 \%$ vs large $6.49 \%$, $\mathrm{p}=0.2714$ ) and discharge to home frequency (small $36.08 \%$ vs medium $41.24 \%$ vs large $38.92 \%, p=0.0875$ ) did not significantly differ by the size of the health system (Table 4).

\section{Discussion}

In a nationwide private health insurance database, we found significant increases in the proportional usage of MT, IV tPA, and combination MT and IV tPA from 2012 to 2018. In addition, we found substantial changes in hospital discharge patterns, most notably significant decreases in routine discharge home and transfers to other hospitals, and significant increases in discharge to home health and inpatient rehabilitation facilities. Most strikingly, we found a significant decrease in mortality at all time points during the same period. This improvement in mortality was primarily in older patients, with no significant trends in age-standardized mortality in those aged 18 to 70 years. The most important advancement in the last decade in 
TABLE 3. Standardized outcomes of patients with AIS by treatment

\begin{tabular}{|c|c|c|c|c|c|c|c|c|}
\hline \multirow[b]{2}{*}{ Characteristic } & \multicolumn{7}{|c|}{ No. of Patients (\%) } & \multirow[b]{2}{*}{ p Value } \\
\hline & $\begin{array}{c}2012 \\
(n=12,831)\end{array}$ & $\begin{array}{c}2013 \\
(n=13,646)\end{array}$ & $\begin{array}{c}2014 \\
(n=13,898)\end{array}$ & $\begin{array}{c}2015 \\
(n=15,474)\end{array}$ & $\begin{array}{c}2016 \\
(n=18,227)\end{array}$ & $\begin{array}{c}2017 \\
(n=21,826)\end{array}$ & $\begin{array}{c}2018 \\
(n=21,932)\end{array}$ & \\
\hline MT & $201(1.6)$ & $212(1.6)$ & $242(1.7)$ & $432(2.8)$ & $594(3.3)$ & $934(4.3)$ & $1,248(5.7)$ & \\
\hline HT & $63(31.3)$ & $59(27.8)$ & $64(26.5)$ & $100(22.2)$ & $161(23.6)$ & $243(24.1)$ & $319(22.4)$ & 0.0291 \\
\hline MCE & $54(26.9)$ & $43(20.3)$ & $52(21.5)$ & $97(21.5)$ & $158(23.2)$ & $221(22.0)$ & $284(20.9)$ & 0.2506 \\
\hline \multicolumn{9}{|l|}{ Discharge disposition } \\
\hline Home routine & $34(16.9)$ & $35(16.5)$ & $52(21.5)$ & $91(20.2)$ & $164(24.1)$ & $215(21.4)$ & $297(21.8)$ & 0.0686 \\
\hline Home health & $4(2.0)$ & $11(5.2)$ & $11(4.6)$ & $26(5.8)$ & $49(7.2)$ & $68(6.8)$ & $115(8.5)$ & 0.0001 \\
\hline Skilled nursing facility & $34(16.9)$ & $41(19.3)$ & $46(19.0)$ & $93(20.6)$ & $120(17.6)$ & $191(19.0)$ & $252(18.5)$ & 0.9598 \\
\hline $\begin{array}{l}\text { Transfer to short-term } \\
\text { hospital }\end{array}$ & $28(13.9)$ & $31(14.6)$ & $26(10.7)$ & $42(9.3)$ & $60(8.8)$ & $77(7.7)$ & $117(8.6)$ & 0.0005 \\
\hline Inpatient rehabilitation & $38(18.9)$ & $46(21.7)$ & $47(19.4)$ & $103(22.8)$ & $131(19.2)$ & $194(19.3)$ & $303(22.3)$ & 0.4799 \\
\hline Other† & $63(31.3)$ & $48(22.6)$ & $60(24.8)$ & $96(21.3)$ & $157(23.1)$ & $262(26.0)$ & $277(20.4)$ & 0.0202 \\
\hline \multicolumn{9}{|l|}{ Mortality } \\
\hline 30-day & $33(16.4)$ & $19(9.0)$ & $20(8.3)$ & $53(11.8)$ & $65(9.5)$ & $103(10.2)$ & $123(9.0)$ & 0.0415 \\
\hline 60-day & $43(21.4)$ & $31(14.6)$ & 34 (14.1) & $73(16.2)$ & $98(14.4)$ & $149(14.8)$ & $179(13.2)$ & 0.0144 \\
\hline 90-day & $43(21.4)$ & $31(14.6)$ & $35(14.5)$ & 80 (17.7) & $107(15.7)$ & $159(15.8)$ & $192(14.1)$ & 0.0414 \\
\hline $6-\mathrm{mo}$ & $45(22.4)$ & $42(19.8)$ & $37(15.3)$ & $86(19.1)$ & $115(16.9)$ & $170(16.9)$ & $209(15.4)$ & 0.0106 \\
\hline IV tPA & $808(6.3)$ & $995(7.3)$ & $1,060(7.6)$ & $1,347(8.7)$ & $1,861(10.2)$ & $2,319(10.6)$ & $2,586(11.8)$ & \\
\hline HT & $100(12.4)$ & $135(13.6)$ & $128(12.1)$ & $148(11.0)$ & $215(11.6)$ & $272(11.7)$ & $325(12.6)$ & 0.7268 \\
\hline MCE & $69(8.5)$ & $87(8.7)$ & $114(10.8)$ & $120(8.9)$ & $137(7.4)$ & $182(7.9)$ & $210(8.1)$ & 0.0717 \\
\hline \multicolumn{9}{|l|}{ Discharge disposition } \\
\hline Home routine & $296(36.6)$ & $387(38.9)$ & $403(38.0)$ & $517(38.4)$ & $742(39.9)$ & $917(39.5)$ & $1,037(40.1)$ & 0.0614 \\
\hline Home health & $52(6.4)$ & $74(7.4)$ & $102(9.6)$ & $122(9.1)$ & $181(9.7)$ & $241(10.4)$ & $325(12.6)$ & $<0.0001$ \\
\hline Skilled nursing facility & $146(18.1)$ & $133(13.4)$ & $153(14.4)$ & $206(15.3)$ & $302(16.2)$ & $372(16.0)$ & $386(14.9)$ & 0.0308 \\
\hline $\begin{array}{l}\text { Transfer to short-term } \\
\text { hospital }\end{array}$ & $84(10.4)$ & $119(12.0)$ & $95(9.0)$ & $100(7.4)$ & $101(5.4)$ & $132(5.7)$ & $135(5.2)$ & 0.0878 \\
\hline Inpatient rehabilitation & $91(11.3)$ & $124(12.5)$ & $133(12.6)$ & $222(16.5)$ & $273(14.7)$ & $323(13.9)$ & $378(14.6)$ & 0.0183 \\
\hline Other† & $139(17.2)$ & $158(15.9)$ & $174(16.4)$ & $180(13.4)$ & $262(14.1)$ & $334(14.4)$ & $325(12.6)$ & 0.0002 \\
\hline \multicolumn{9}{|l|}{ Mortality } \\
\hline 30-day & $69(8.5)$ & $71(7.1)$ & $80(7.6)$ & $79(5.9)$ & $116(6.2)$ & $139(6.0)$ & $127(4.9)$ & $<0.0001$ \\
\hline 60 -day & $92(11.4)$ & $100(10.1)$ & $103(9.7)$ & $121(9.0)$ & $158(8.5)$ & $192(8.3)$ & $165(6.4)$ & $<0.0001$ \\
\hline 90-day & $95(11.8)$ & $106(10.7)$ & $107(10.1)$ & $131(9.7)$ & $168(9.0)$ & $208(9.0)$ & $181(7.0)$ & $<0.0001$ \\
\hline $6-\mathrm{mo}$ & $107(13.2)$ & $124(12.5)$ & $120(11.3)$ & $145(10.8)$ & $189(10.2)$ & $237(10.2)$ & $198(7.7)$ & $<0.0001$ \\
\hline IV tPA and MT & $88(0.6)$ & $95(0.6)$ & $100(0.6)$ & $150(0.8)$ & $176(0.8)$ & $272(1.0)$ & $382(1.4)$ & \\
\hline HT & $25(29.8)$ & $27(29.7)$ & $18(19.2)$ & $37(25.2)$ & $36(21.6)$ & $65(25.5)$ & $82(22.5)$ & 0.2189 \\
\hline MCE & $19(22.6)$ & $20(22.0)$ & $22(23.4)$ & $32(21.8)$ & $37(22.2)$ & $55(21.6)$ & $64(17.5)$ & 0.1630 \\
\hline \multicolumn{9}{|l|}{ Discharge disposition } \\
\hline Home routine & $20(23.8)$ & $17(18.7)$ & $24(25.5)$ & $35(23.8)$ & $43(25.8)$ & $60(23.5)$ & $82(22.5)$ & 0.9880 \\
\hline Home health & $3(3.6)$ & $4(4.4)$ & $3(3.2)$ & $9(6.1)$ & $10(6.0)$ & $13(5.1)$ & $35(9.6)$ & 0.0108 \\
\hline Skilled nursing facility & $12(14.3)$ & $16(17.7)$ & $15(16.0)$ & $29(19.7)$ & $26(15.6)$ & $45(17.7)$ & $68(18.6)$ & 0.4559 \\
\hline $\begin{array}{l}\text { Transfer to short-term } \\
\text { hospital }\end{array}$ & $12(14.3)$ & $18(19.8)$ & $13(13.8)$ & $12(8.2)$ & $9(5.4)$ & $17(6.7)$ & $27(7.4)$ & 0.0001 \\
\hline Inpatient rehabilitation & $14(16.7)$ & $20(22.0)$ & $16(17.0)$ & $36(24.5)$ & $46(27.5)$ & $52(20.4)$ & $87(23.8)$ & 0.2370 \\
\hline Other† & $23(27.4)$ & $16(17.6)$ & $23(24.5)$ & $26(17.7)$ & $33(19.8)$ & $68(26.7)$ & 66 (18.1) & 0.4453 \\
\hline \multicolumn{9}{|l|}{ Mortality } \\
\hline 30-day & $11(13.1)$ & $6(6.6)$ & $5(5.3)$ & $11(7.5)$ & $15(9.0)$ & $32(12.6)$ & $30(8.2)$ & 0.7836 \\
\hline 60-day & $13(15.5)$ & $13(14.3)$ & $8(8.5)$ & $17(11.6)$ & $21(12.6)$ & $44(17.3)$ & $41(11.2)$ & 0.8808 \\
\hline 90-day & $13(15.5)$ & $13(14.3)$ & $8(8.5)$ & $18(12.2)$ & $22(13.2)$ & $46(18.0)$ & $45(12.3)$ & 0.8137 \\
\hline
\end{tabular}


» CONTINUED FROM PAGE 5

TABLE 3. Standardized outcomes of patients with AIS by treatment

\begin{tabular}{|c|c|c|c|c|c|c|c|c|}
\hline \multirow[b]{2}{*}{ Characteristic } & \multicolumn{7}{|c|}{ No. of Patients (\%) } & \multirow[b]{2}{*}{ p Value* } \\
\hline & $\begin{array}{c}2012 \\
(n=12,831)\end{array}$ & $\begin{array}{c}2013 \\
(n=13,646)\end{array}$ & $\begin{array}{c}2014 \\
(n=13,898)\end{array}$ & $\begin{array}{c}2015 \\
(n=15,474)\end{array}$ & $\begin{array}{c}2016 \\
(n=18,227)\end{array}$ & $\begin{array}{c}2017 \\
(n=21,826)\end{array}$ & $\begin{array}{c}2018 \\
(n=21,932)\end{array}$ & \\
\hline \multicolumn{9}{|l|}{ IV tPA and MT (continued) } \\
\hline \multicolumn{9}{|l|}{ Mortality (continued) } \\
\hline 6-mo & $14(16.7)$ & $20(22.0)$ & $8(8.5)$ & $18(12.2)$ & $23(13.8)$ & $48(18.8)$ & 48 (13.2) & 0.6082 \\
\hline Neither MT nor tPA & $11,906(92.8)$ & $12,530(91.8)$ & $12,690(91.3)$ & $13,823(89.3)$ & $15,852(87.0)$ & $18,755(85.9)$ & $18,350(83.7)$ & \\
\hline HT & $539(4.5)$ & $597(4.8)$ & $614(4.8)$ & $656(4.8)$ & $676(4.3)$ & $876(4.7)$ & $941(5.1)$ & 0.1908 \\
\hline MCE & $392(3.3)$ & $477(3.8)$ & $524(4.1)$ & $590(4.3)$ & $712(4.5)$ & $890(4.8)$ & $985(5.4)$ & $<0.0001$ \\
\hline \multicolumn{9}{|l|}{ Discharge disposition } \\
\hline Home routine & $4,996(58.0)$ & $5,147(41.1)$ & $5,028(39.6)$ & $5,554(40.2)$ & $6,201(39.1)$ & $7,161(38.2)$ & $6,928(37.8)$ & $<0.0001$ \\
\hline Home health & $1,280(10.8)$ & $1,464(11.7)$ & $1,416(11.2)$ & $1,485(10.7)$ & $1,898(12.0)$ & $2,407(12.8)$ & $2,406(13.1)$ & $<0.0001$ \\
\hline Skilled nursing facility & $2,426(20.4)$ & $2,506(20.0)$ & $2,706(21.3)$ & $2,797(20.2)$ & $3,190(20.1)$ & $3,924(20.9)$ & $3,733(20.3)$ & 0.7470 \\
\hline $\begin{array}{l}\text { Transfer to short-term } \\
\text { hospital }\end{array}$ & $819(6.9)$ & $752(6.0)$ & $751(5.9)$ & $882(6.4)$ & $911(5.8)$ & $936(5.0)$ & $907(4.9)$ & $<0.0001$ \\
\hline Inpatient rehabilitation & $1,023(8.6)$ & $1,156(9.2)$ & $1,300(10.2)$ & $1,486(10.8)$ & $1,879(11.9)$ & $2,239(11.9)$ & 2,307 (12.6) & $<0.0001$ \\
\hline Other† & $1,362(11.4)$ & $1,505(12.0)$ & $1,489(11.7)$ & $1,619(11.7)$ & $1,773(11.2)$ & 2,088 (11.1) & $2,069(11.3)$ & 0.0348 \\
\hline \multicolumn{9}{|l|}{ Mortality } \\
\hline 30-day & $527(4.4)$ & $498(4.0)$ & $568(4.5)$ & $635(4.6)$ & $564(3.6)$ & $677(3.6)$ & $696(3.8)$ & $<0.0001$ \\
\hline 60-day & $860(7.2)$ & $800(6.4)$ & $886(7.0)$ & $960(6.9)$ & $962(6.1)$ & $1,079(5.8)$ & $1,015(5.5)$ & $<0.0001$ \\
\hline 90-day & $992(8.3)$ & $897(7.2)$ & $1,002(7.9)$ & $1,092(7.9)$ & $1,095(6.9)$ & $1,211(6.5)$ & $1,147(6.3)$ & $<0.0001$ \\
\hline 6-mo & $1,183(9.9)$ & $1,066(8.5)$ & $1,208(9.5)$ & $1,294(9.4)$ & $1,319(8.3)$ & $1,481(7.9)$ & $1,341(7.3)$ & $<0.0001$ \\
\hline
\end{tabular}

Boldface type indicates statistical significance.

${ }^{*}$ Cochran-Armitage test.

† Includes intermediate care facility, other type of institution for inpatient care, left against medical advice, admitted as inpatient to this hospital, still inpatient expecting to return as outpatient or status yet to be defined at the state level, transfer to a federal healthcare facility, hospice (home or medical), transfer within the institution, and transfer to a long-term hospital, nursing home facility certified under Medicare, psychiatric hospital, critical access hospital, or another type of healthcare facility.

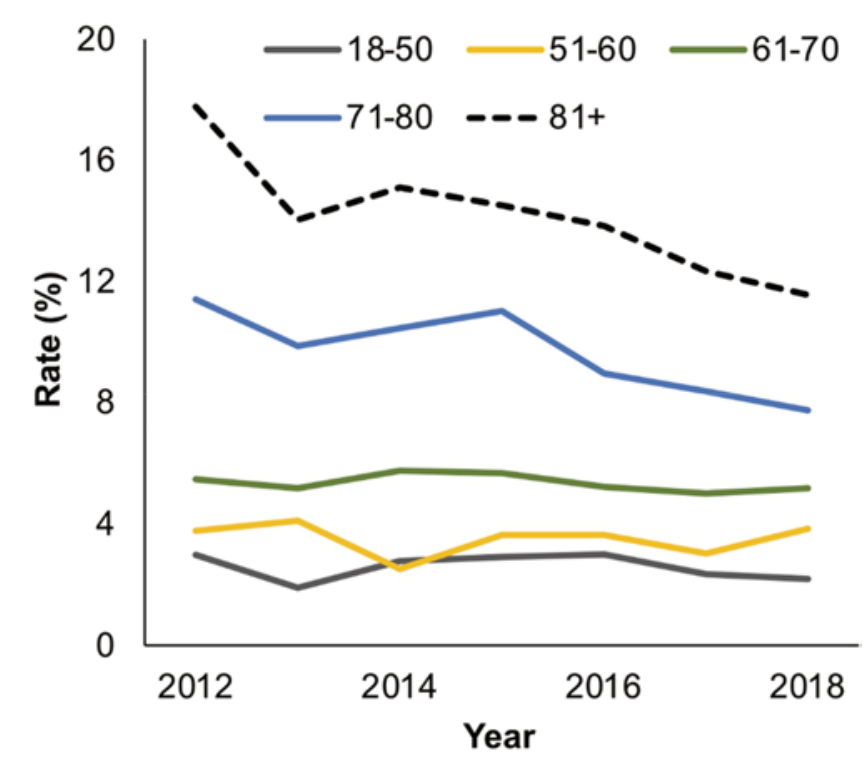

FIG. 3. Age-standardized 90-day mortality. Age groups are in years.
AIS care has been the introduction and continued development of MT. The use of MT increased significantly after the results of landmark trials., ${ }^{71,26,27}$ By demonstrating increased use of MT over time at the population level, using large-scale clinical data from 2012 to 2018, we corroborated and updated previously published trends in increasing MT usage through 2016 and found that these trends continued to increase through 2018. ${ }^{16,23}$ Furthermore, we identified that the largest increase in MT rates was between 2017 and 2018, possibly illustrating the influence of the late-window trials published in early $2018 .{ }^{28,29}$ The decreased mortality rate we reported following MT treatment in 2018 compared with 2012 is particularly noteworthy. Although we cannot directly identify causes of decreasing mortality, it is likely that this at least partially reflects improvements in MT, such as superior patient selection, device, and procedural learning curves, as well as postthrombectomy care. Additionally, broadened indications for MT may mean patients with less severe strokes are receiving MT, with a resultant lower risk of mortality.

Nevertheless, the improvements we report in MT and posthrombectomy care alone are likely insufficient to account for the overall decrease in mortality after AIS. Increasing awareness and organization of stroke care likely plays a major role in explaining the increased treatment rates and improved outcomes found in our study among 
TABLE 4. Outcomes of patients with AIS by treatment and hospital size

\begin{tabular}{|c|c|c|c|c|}
\hline \multirow[b]{2}{*}{ Characteristics } & \multicolumn{3}{|c|}{ No. of Patients (\%) } & \multirow[b]{2}{*}{$p$ Value $^{*}$} \\
\hline & Large (>249 beds) & Medium (101-249 beds) & Small (<101 beds) & \\
\hline All patients & 60,019 & 25,296 & 7,906 & \\
\hline \multicolumn{5}{|l|}{ Discharge disposition } \\
\hline Home routine & $23,588(39.90)$ & $10,091(39.89)$ & $2,892(36.58)$ & $<0.0001$ \\
\hline Home health & $6,780(11.30)$ & $3,012(11.91)$ & $939(11.88)$ & 0.0218 \\
\hline Skilled nursing facility & $11,438(19.06)$ & $5,263(20.81)$ & $1,799(22.75)$ & $<0.0001$ \\
\hline Transfer to short-term hospital & $3,364(5.60)$ & $1,614(6.38)$ & $646(8.17)$ & $<0.0001$ \\
\hline Inpatient rehabilitation & $7,772(12.95)$ & $2,580(10.20)$ & $611(7.73)$ & $<0.0001$ \\
\hline Other† & 7,077 (11.79) & $2,736(10.82)$ & $1,019(12.89)$ & $<0.0001$ \\
\hline \multicolumn{5}{|l|}{ Mortality } \\
\hline 30-day & $2,694(4.49)$ & $1,002(3.96)$ & $345(4.36)$ & 0.0025 \\
\hline 60-day & $4,173(6.95)$ & $1,508(5.96)$ & $522(6.60)$ & $<0.0001$ \\
\hline 90-day & $4,684(7.80)$ & $1,715(6.78)$ & $590(7.46)$ & $<0.0001$ \\
\hline $6-\mathrm{mo}$ & $5,475(9.12)$ & $2,075(8.20)$ & $712(9.01)$ & $<0.0001$ \\
\hline MT & 2,799 & 399 & 31 & \\
\hline \multicolumn{5}{|l|}{ Discharge disposition } \\
\hline Home routine & $609(21.76)$ & $97(24.31)$ & $3(9.68)$ & 0.1299 \\
\hline Home health & $187(6.68)$ & $23(5.76)$ & $7(22.58)$ & 0.0015 \\
\hline Skilled nursing facility & $491(17.54)$ & $94(23.56)$ & $5(16.13)$ & 0.0138 \\
\hline Transfer to short-term hospital & $254(9.07)$ & $45(11.28)$ & $1(3.23)$ & 0.1847 \\
\hline Inpatient rehabilitation & $628(22.44)$ & $50(12.53)$ & $7(22.58)$ & $<0.0001$ \\
\hline Other† & $630(22.51)$ & $90(22.56)$ & $8(25.81)$ & 0.9089 \\
\hline \multicolumn{5}{|l|}{ Mortality } \\
\hline 30-day & $277(9.90)$ & $39(9.77)$ & $7(22.58)$ & 0.0637 \\
\hline 60-day & $410(14.65)$ & $55(13.78)$ & $8(25.81)$ & 0.1897 \\
\hline 90-day & $435(15.54)$ & $59(14.79)$ & $8(25.81)$ & 0.2644 \\
\hline $6-\mathrm{mo}$ & $476(17.01)$ & $62(15.54)$ & $8(25.81)$ & 0.3169 \\
\hline IV tPA & 6,547 & 1,974 & 316 & \\
\hline \multicolumn{5}{|l|}{ Discharge disposition } \\
\hline Home routine & $2,548(38.92)$ & $814(41.24)$ & $114(36.08)$ & 0.0875 \\
\hline Home health & $619(9.45)$ & $198(10.03)$ & $41(12.97)$ & 0.1023 \\
\hline Skilled nursing facility & $992(15.15)$ & $329(16.67)$ & $47(14.87)$ & 0.2526 \\
\hline Transfer to short-term hospital & $440(6.72)$ & $162(8.21)$ & $26(8.23)$ & 0.0579 \\
\hline Inpatient rehabilitation & 963 (14.71) & $213(10.79)$ & $50(15.82)$ & $<0.0001$ \\
\hline Other† & $985(15.05)$ & $258(13.07)$ & $38(12.03)$ & 0.0410 \\
\hline \multicolumn{5}{|l|}{ Mortality } \\
\hline 30-day & $425(6.49)$ & $110(5.57)$ & $17(5.38)$ & 0.2714 \\
\hline 60-day & $589(9.00)$ & $144(7.29)$ & $22(6.96)$ & 0.0356 \\
\hline 90-day & $629(9.61)$ & $158(8.00)$ & $23(7.28)$ & 0.0477 \\
\hline $6-\mathrm{mo}$ & $699(10.68)$ & $180(9.12)$ & $25(7.91)$ & 0.0516 \\
\hline
\end{tabular}

Boldface type indicates statistical significance.

* Cochran-Armitage test.

$\dagger$ Includes intermediate care facility, other type of institution for inpatient care, left against medical advice, admitted as inpatient to this hospital, still inpatient expecting to return as outpatient or status yet to be defined at the state level, transfer to a federal healthcare facility, hospice (home or medical), transfer within the institution, and transfer to a long-term hospital, nursing home facility certified under Medicare, psychiatric hospital, critical access hospital, or another type of healthcare facility.

all treatment groups. This is evidenced by the fact that primary stroke centers (PSCs), first established in 2004, have been associated with improved outcomes, including a $14 \%$ decrease in death, $18 \%$ decrease in death or institutionalized care, and $18 \%$ decrease in death or dependency. ${ }^{14,30-32}$
By late 2010, an estimated $53 \%$ of the US population had adequate access to a PSC, and the number of PSCs has continued to increase since then. In 2012, the first CSCs were certified and have since been found to have higher MT rates, shorter door-to-IV tPA time, and shorter door-to-MT 
time than PSCs. ${ }^{33}$ It is possible that the decreased rate of transfers in recent years seen in our data set reflects the increased accessibility of PSCs and CSCs across the country.

Alternatively, the improved mortality rates may reflect differences in hospital presentation with mild stroke, differences in the diagnosis of mild stroke, or changes in admission/discharge or insurance coding practices. For example, it is possible that practitioners are admitting patients with milder strokes or transient ischemic attacks and classifying the conditions as AIS. This may contribute to the considerable increase in patients with AIS identified in our database from 2012 to 2018, against a backdrop of putatively declining stroke incidence. It is also possible that practitioners are withdrawing care less frequently and potentially artificially deflating the mortality rate. Another possibility is that our entire study population has become a healthier population in recent years, contributing to better outcomes. Further investigations are necessary to assess the plausibility of these and other possible explanations.

Despite these advancements in stroke treatment, organized stroke care, and patient education, stroke remains the second leading cause of death in the world., ${ }^{1,2}$ Stroke mortality has steadily decreased over the last few decades; however, studies have found that these declines in stroke mortality may be slowing or even reversing. ${ }^{15,21}$ Hall et al. ${ }^{34}$ suggested this stagnation in stroke mortality may be secondary to an increasing mortality in middle-aged (35-64 years) adults with ischemic stroke. While these changes are largely understood to represent declining stroke incidence, it is possible that declining case fatality may also be contributing. Fitting with the overall trends in stroke mortality, our data show a reduction in overall case fatality, no change in case fatality among patients younger than 70 years of age hospitalized with stroke, but a significant reduction in case fatality in those older than 70 years.

Our study also found that medium-sized hospitals were associated with the lowest mortality rates in patients with AIS. While statistically significant, the clinical relevance of this finding is questionable, as the sample sizes were large and the absolute difference in mortality rates was less than $1 \%$. It is possible that this difference in mortality reflects the expansion and subsequent benefit of stroke units into smaller community hospitals in recent years. ${ }^{35}$ Another possibility is that "large" hospitals represent larger tertiary care centers with the sickest stroke patients in our cohort, whereas "small" hospitals likely transfer many stroke patients to other centers for care, possibly excepting some devastating strokes beyond treatment, both of which would contribute to higher mortality rates. Moreover, we suspect that no differences were seen in mortality in patients treated with MT likely because of the small sample size of those patients in general and especially at small hospitals. Given the trend toward significance of worse outcomes among patients treated with MT at small hospitals compared with large health systems (30-day mortality $22.6 \%$ vs $9.9 \%, \mathrm{p}=0.0637$; and disposition to home $9.7 \%$ vs $21.8 \%, p=0.1299$ ), as more data are collected on these parameters we would expect a clearer relationship between hospital size and outcomes following MT.

\section{Limitations and Generalizability}

The shortcomings of administrative data in general in stroke outcomes research have been well described. ${ }^{36,37}$ Most prominently, stroke severity is not reflected in billing data, and functional outcomes after stroke are difficult to measure. Thus, we were unable to correlate our mortality data to individual patient stroke severity. Similarly, the database does not include hospital information such as the location or type of hospital or, importantly, stroke center status. Billing classification of a stroke diagnosis also introduces uncertainty for mild strokes, as those classified as having a primary diagnosis of ischemic stroke may vary between institutions and over time. Coding for treatments, including MT and tPA, however, is less ambiguous. The inclusion of more mild strokes under the stroke primary diagnosis codes later in the study could account for some of the improvement seen in mortality but would also be expected to lower MT and tPA treatment rates, when in fact the opposite was seen. A combination of these effects cannot be excluded.

Another major shortcoming of using administrative data is the inability to capture functional outcomes such as modified Rankin Scale (mRS) scores. To estimate functional status using large databases, authors generally use discharge disposition as a surrogate for the mRS score. One study found significant correlations between discharge dispositions and mRS scores. ${ }^{38}$ Specifically, its authors found discharge to nursing home and rehabilitation facilities to be predictive of unfavorable outcome (mRS scores 2-6) and discharge home to be predictive of favorable outcome $(\mathrm{mRS}$ scores $<2)$. While data sets such as registries of more granular information such as mRS scores are certainly desirable, discharge disposition currently provides valuable information on functional status in poststroke patients.

The generalizability of our results is limited, as the study was restricted to a privately insured population. This included both those with private insurance only and those with a private insurance supplement to Medicare. In 2018, roughly only two-thirds of Americans had private health insurance. Furthermore, insurance status plays a significant role in ischemic stroke outcomes and time to presentation for treatment, likely because uninsured and Medicare/ Medicaid patients generally have a higher number of comorbidities and risk factors for stroke. ${ }^{39}$ While having only privately insured patients does control somewhat for variation, we would also expect these patients to be healthier compared with the overall US patient population, and this may be reflected in decreased mortality rates and increased treatment rates.

\section{Conclusions}

During the study period, the use of tPA and MT for AIS significantly increased while mortality following AIS significantly decreased. The most dramatic decrease in mortality was observed in the population of patients treated with MT, followed by the IV tPA treatment and notreatment groups. Trends in age-standardized mortality revealed a significant decrease in mortality in older patients ( $\geq 71$ years) with AIS and no significant trends in mortality in younger patients (18-70 years) with AIS. Further inves- 
tigation is necessary to identify the most significant factors contributing to these trends.

\section{References}

1. GBD 2016 Stroke Collaborators. Global, regional, and national burden of stroke, 1990-2016: a systematic analysis for the Global Burden of Disease Study 2016. Lancet Neurol. 2019; 18(5):439-458.

2. Guzik A, Bushnell C. Stroke epidemiology and risk factor management. Continuum (Minneap Minn). 2017;23(1, Cerebrovascular Disease):15-39.

3. Emberson J, Lees KR, Lyden P, et al. Effect of treatment delay, age, and stroke severity on the effects of intravenous thrombolysis with alteplase for acute ischaemic stroke: a meta-analysis of individual patient data from randomised trials. Lancet. 2014;384(9958):1929-1935.

4. National Institute of Neurological Disorders and Stroke rt-PA Stroke Study Group. Tissue plasminogen activator for acute ischemic stroke. N Engl J Med. 1995;333(24):1581-1587.

5. Damani R. A brief history of acute stroke care. Aging (Albany NY). 2018;10(8):1797-1798.

6. Bracard S, Ducrocq X, Mas JL, et al. Mechanical thrombectomy after intravenous alteplase versus alteplase alone after stroke (THRACE): a randomised controlled trial. Lancet Neurol. 2016;15(11):1138-1147.

7. Campbell BC, Mitchell PJ, Kleinig TJ, et al. Endovascular therapy for ischemic stroke with perfusion-imaging selection. N Engl J Med. 2015;372(11):1009-1018.

8. Goyal M, Demchuk AM, Menon BK, et al. Randomized assessment of rapid endovascular treatment of ischemic stroke. N Engl J Med. 2015;372(11):1019-1030.

9. Goyal M, Menon BK, van Zwam WH, et al. Endovascular thrombectomy after large-vessel ischaemic stroke: a metaanalysis of individual patient data from five randomised trials. Lancet. 2016;387(10029):1723-1731.

10. Jovin TG, Chamorro A, Cobo E, et al. Thrombectomy within 8 hours after symptom onset in ischemic stroke. $N$ Engl J Med. 2015;372(24):2296-2306.

11. Saver JL, Goyal M, Bonafe A, et al. Stent-retriever thrombectomy after intravenous t-PA vs. t-PA alone in stroke. $N$ Engl J Med. 2015;372(24):2285-2295.

12. Hirsch JA, Yoo AJ, Nogueira RG, et al. Case volumes of intra-arterial and intravenous treatment of ischemic stroke in the USA. J Neurointerv Surg. 2009;1(1):27-31.

13. Stein L, Tuhrim S, Fifi J, et al. National trends in endovascular therapy for acute ischemic stroke: utilization and outcomes. J Neurointerv Surg. 2020;12(4):356-362.

14. Gorelick PB. Primary and comprehensive stroke centers: history, value and certification criteria. J Stroke. 2013;15(2):78-89.

15. Koton S, Schneider AL, Rosamond WD, et al. Stroke incidence and mortality trends in US communities, 1987 to 2011. JAMA. 2014;312(3):259-268.

16. Villwock MR, Padalino DJ, Deshaies EM. Trends in mortality following mechanical thrombectomy for the treatment of acute ischemic stroke in the USA. J Neurointerv Surg. 2016; 8(5):457-460.

17. Ovbiagele B. Nationwide trends in in-hospital mortality among patients with stroke. Stroke. 2010;41(8):1748-1754.

18. Ramirez L, Kim-Tenser MA, Sanossian N, et al. Trends in acute ischemic stroke hospitalizations in the United States. $J$ Am Heart Assoc. 2016;5(5): 003233.

19. Smith EE, Saver JL, Cox M, et al. Increase in endovascular therapy in Get With The Guidelines-Stroke after the publication of pivotal trials. Circulation. 2017;136(24):2303-2310.

20. Yafasova A, Fosbøl EL, Christiansen MN, et al. Time trends in incidence, comorbidity, and mortality of ischemic stroke in Denmark (1996-2016). Neurology. 2020;95(17):e2343-e2353.

21. Yang Q, Tong X, Schieb L, et al. Vital signs: recent trends in stroke death rates-United States, 2000-2015. MMWR Morb Mortal Wkly Rep. 2017;66(35):933-939.

22. Hassan AE, Chaudhry SA, Grigoryan M, et al. National trends in utilization and outcomes of endovascular treatment of acute ischemic stroke patients in the mechanical thrombectomy era. Stroke. 2012;43(11):3012-3017.

23. Rumalla K, Ottenhausen M, Kan P, Burkhardt JK. Recent nationwide impact of mechanical thrombectomy on decompressive hemicraniectomy for acute ischemic stroke. Stroke. 2019;50(8):2133-2139.

24. Volny O, Krajina A, Belaskova S, et al. Mechanical thrombectomy performs similarly in real world practice: a 2016 nationwide study from the Czech Republic. J Neurointerv Surg. 2018;10(8):741-745.

25. Ekker MS, Verhoeven JI, Vaartjes I, et al. Stroke incidence in young adults according to age, subtype, sex, and time trends. Neurology. 2019;92(21):e2444-e2454.

26. Demchuk AM, Goyal M, Menon BK, et al. Endovascular treatment for Small Core and Anterior circulation Proximal occlusion with Emphasis on minimizing CT to recanalization times (ESCAPE) trial: methodology. Int J Stroke. 2015;10(3):429-438.

27. Fransen PS, Beumer D, Berkhemer OA, et al. MR CLEAN, a multicenter randomized clinical trial of endovascular treatment for acute ischemic stroke in the Netherlands: study protocol for a randomized controlled trial. Trials. 2014;15:343.

28. Nogueira RG, Jadhav AP, Haussen DC, et al. Thrombectomy 6 to 24 hours after stroke with a mismatch between deficit and infarct. N Engl J Med. 2018;378(1):11-21.

29. Albers GW, Marks MP, Kemp S, et al. Thrombectomy for stroke at 6 to 16 hours with selection by perfusion imaging. $N$ Engl J Med. 2018;378(8):708-718.

30. Kidwell CS, Shephard T, Tonn S, et al. Establishment of primary stroke centers: a survey of physician attitudes and hospital resources. Neurology. 2003;60(9):1452-1456.

31. Saposnik G, Fang J, O'Donnell M, et al. Escalating levels of access to in-hospital care and stroke mortality. Stroke. 2008; 39(9):2522-2530.

32. Xian Y, Holloway RG, Chan PS, et al. Association between stroke center hospitalization for acute ischemic stroke and mortality. JAMA. 2011;305(4):373-380.

33. Man S, Zhao X, Uchino K, et al. Comparison of acute ischemic stroke care and outcomes between comprehensive stroke centers and primary stroke centers in the United States. Circ Cardiovasc Qual Outcomes. 2018;11(6):e004512.

34. Hall EW, Vaughan AS, Ritchey MD, et al. Stagnating national declines in stroke mortality mask widespread county-level increases, 2010-2016. Stroke. 2019;50(12):3355-3359.

35. Tamm A, Siddiqui M, Shuaib A, et al. Impact of stroke care unit on patient outcomes in a community hospital. Stroke. 2014;45(1):211-216.

36. Kestle JR. Administrative database research. J Neurosurg. 2015;122(2):441-442.

37. Ung D, Kim J, Thrift AG, et al. Promising use of big data to increase the efficiency and comprehensiveness of stroke outcomes research. Stroke. 2019;50(5):1302-1309.

38. Qureshi AI, Chaudhry SA, Sapkota BL, et al. Discharge destination as a surrogate for Modified Rankin Scale defined outcomes at 3- and 12-months poststroke among stroke survivors. Arch Phys Med Rehabil. 2012;93(8):1408-1413.e1.

39. Medford-Davis LN, Fonarow GC, Bhatt DL, et al. Impact of insurance status on outcomes and use of rehabilitation services in acute ischemic stroke: findings from Get With The Guidelines-Stroke. J Am Heart Assoc. 2016;5(11):e004282.

\section{Disclosures}

The authors report no conflict of interest concerning the materials or methods used in this study or the findings specified in this paper. 


\section{Author Contributions}

Conception and design: Pandey, Anand, Benjamin, Park,

Wilkinson, Daou. Acquisition of data: Pandey, Anand, Benjamin, Park, Wilkinson, Daou. Analysis and interpretation of data: all authors. Drafting the article: all authors. Critically revising the article: Pandey, Anand, Wilkinson, Daou, Burke. Reviewed submitted version of manuscript: all authors. Approved the final version of the manuscript on behalf of all authors: Pandey. Statistical analysis: Benjamin, Adapa, Park, Wilkinson, Daou. Study supervision: Pandey, Wilkinson, Daou, Burke.

\section{Supplemental Information}

Online-Only Content

Supplemental material is available online.

Supplemental Tables 1 and 2. https://thejns.org/doi/suppl/ 10.3171/2021.4.FOCUS21117.

\section{Correspondence}

Aditya S. Pandey: University of Michigan, Ann Arbor, MI. adityap@med.umich.edu. 